\title{
Physical Activity and Cognitive Function among Older Adults with an Elevated Gamma Gap
}

\author{
Emily Frith ${ }^{a}$ Paul D. Loprinzi ${ }^{b}$ \\ a Physical Activity Epidemiology Laboratory, Exercise Psychology Laboratory, The University of Mississipi, \\ University, MS, USA; ${ }^{b}$ Jackson Heart Study Vanguard Center of Oxford, Physical Activity Epidemiology \\ Laboratory, Department of Health, Exercise Science, and Recreation Management, The University of Mississippi, \\ University, MS, USA
}

\section{Significance of the Study}

- This novel study demonstrates that an elevated gamma gap is associated with worse cognition, and that among subjects with an elevated gamma gap, those who are physically active have greater cognition.

\section{Keywords}

Cognition · Elderly · Epidemiology · Exercise

\begin{abstract}
Objective: An elevated gamma gap is indicative of high serum concentrations of globulin proteins, some of which elicit acute inflammatory responses. An impaired cognitive function has been linked to central and peripheral inflammation, while exercise is associated with protective, anti-inflammatory benefits. In this study, we evaluated whether the gamma gap is associated with cognitive function among older adults and whether physical activity is favorably associated with cognitive function among those with an elevated gamma gap. Materials and Methods: Data from the 19992002 National Health and Nutrition Examination Survey (NHANES) were used to identify 2,352 older adults aged between 60 and 85 years. The gamma gap was evaluated by subtracting albumin from total protein, i.e., gamma gap =
\end{abstract}

\begin{tabular}{ll}
\hline KARGER & $\begin{array}{l}\text { (c) } 2018 \text { The Author(s) } \\
\text { Published by S. Karger AG, Basel }\end{array}$ \\
E-Mail karger@karger.com & $\begin{array}{l}\text { This is an Open Access article licensed under the Creative Commons } \\
\text { Attribution-NonCommercial-4.0 International License (CC BY-NC) } \\
\text { (http://www.karger.com/Services/OpenAccessLicense), applicable to } \\
\text { the online version of the article only. Usage and distribution for } \\
\text { commercial purposes requires written permission. }\end{array}$
\end{tabular}

total protein $(\mathrm{g} / \mathrm{dL})$ - albumin $(\mathrm{g} / \mathrm{dL})$. Those at or above 3.1 $\mathrm{g} / \mathrm{dL}(31.0 \mathrm{~g} / \mathrm{L})$ were considered to have an elevated gamma gap. The Digit Symbol Substitution Test (DSST) was used to assess cognitive function tasks of pairing and free recall among participants. Participants were asked open-ended questions about participation in leisure-time physical activity over the previous 30 days. Results: Those with an elevated gamma gap (DSST, 44.8) had a lower cognitive function score when compared to those without an elevated gamma gap (DSST, 50.1) ( $p<0.001)$. After adjustments, and among those with an elevated gamma gap, those meeting the moderate-to-vigorous intensity physical activity (MVPA) guidelines (vs. not meeting them) had a DSST score of 6.42 units higher $(\beta=6.42,95 \% \mathrm{Cl} 3.85-8.99, p<0.001)$. Conclusion: In this national sample of older adults, the gamma gap was associated with cognitive function, and among those with an elevated gamma gap, meeting the physical activity guidelines was associated with a higher cognitive function. Relevant clinical implications are discussed, as the gamma gap

Paul D. Loprinzi

Department of Health, Exercise Science, and Recreation Management The University of Mississippi 229 Turner Center, University, MS 38677 (USA)

E-Mail pdloprin@olemiss.edu 
may be predictive of the risk for early mortality and reduced quality of life. Experimental work is needed to investigate whether physical activity training programs are effective in reducing an elevated gamma gap and preserving optimal cognitive functioning among at-risk individuals.

(C) 2018 The Author(s)

Published by S. Karger AG, Basel

\section{Introduction}

The gamma gap is known as the globulin fraction, an indicator for cardiovascular risk factors [1] associated with morbidity and mortality [2]. Globulins are dissolved plasma proteins, clinically measurable in plasma serum concentrations. The gamma gap is calculated by subtracting albumin $(\mathrm{g} / \mathrm{dL})$ from total plasma protein concentration $(\mathrm{g} / \mathrm{dL})[3]$. The globulin fraction is divided into four separate fractions, $a_{1}, a_{2}, \beta$, and $\gamma$, which are classified according to their migratory sequence during electrophoresis. An increase in plasma globulin proteins results in an elevated globulin fraction, usually due to changes in immunoglobulin content [ 3 ]. $\beta$ and $y$ globulins may be higher in chronic disease states, such as diabetes or heart disease. These globulin proteins are significant determinants of acute inflammatory responses [4]. In addition, pathogenic states characterized by infection, plasma cell malignancy, as well as varied autoimmune disorders that often exhibit elevated immunoglobulin and albumin-independent protein content may all be predictive of an increased gamma gap [5]. An elevated gamma gap has been associated with all-cause mortality in nonclinical populations, with inflammation compromising sufficient albumin production, and subsequently promoting an increased serum C-reactive protein level, a likely mechanism for significant health complications and/or early mortality [5]. An impaired cognitive function has been linked to central and peripheral inflammation [6]. The globulin proteins, characteristic of a gamma gap elevation, are positively associated with inflammation, contributing to the likelihood for those with an elevated gamma gap to be at a higher risk for cognitive dysfunction. Previous work has further detailed and described this gamma gap $[2,7,8]$.

To our knowledge, no study has specifically evaluated the effect of an elevated gamma gap on cognitive function among the elderly. Based on the current understanding of the gamma gap, and its role in inflammatory disease processes $[4,9]$, it is conceivable that those with an elevated gamma gap would have a diminished cognitive function, and that physical activity may offer a protective effect among those with an elevated gamma gap. Exercise is associated with numerous health outcomes [10] and has been shown to elicit protective, anti-inflammatory benefits $[11,12]$, which could potentially have a moderating effect on inflammatory-based cognitive dysfunction in individuals with an elevated gamma gap. Thus, the purpose of this study was to (1) determine whether the gamma gap is associated with cognitive function, and (2) evaluate whether meeting physical activity guidelines (150 min of moderate-to-vigorous intensity physical activity [MVPA] per week) [13] is favorably associated with cognitive function among older adults with an elevated gamma gap. Such an investigation will have important public health implications in identifying the potential putative role that physical activity may play in influencing cognition among older adults with an elevated gamma gap.

\section{Methods}

Study Design

Data from the 1999-2002 National Health and Nutrition Examination Survey (NHANES) were used. Study procedures were approved by the National Center for Health Statistics ethics review board, with informed consent obtained prior to data collection.

The NHANES is an ongoing survey conducted by the Centers for Disease Control and Prevention that uses a representative sample of noninstitutionalized United States civilians selected by a complex, stratified, and clustered probability design. The multistage design consists of 4 stages, including the identification of counties, segments (city blocks), random selection of households within the segments, and random selection of individuals within the households. Further information on NHANES methodology and data collection is available on the NHANES website (http:// www.cdc.gov/nchs/nhanes.htm).

\section{Gamma Gap}

Using the Hitachi Model 704 multichannel analyzer, the gamma gap was evaluated by subtracting albumin (bromocresol purple reagent using the Beckman Synchron LX20) from total protein (colorimetric assay using the Beckman Synchron LX20), i.e., gamma gap $=$ total protein $(\mathrm{g} / \mathrm{dL})-$ albumin $(\mathrm{g} / \mathrm{dL})$. As in previous reports $[2,7,8]$, those at or above $3.1 \mathrm{~g} / \mathrm{dL}(31.0 \mathrm{~g} / \mathrm{L})$ were considered to have an elevated gamma gap.

\section{Cognitive Function}

The Digit Symbol Substitution Test (DSST) was used to assess cognitive function [14-16], which was administered to adults over 60 years of age. The DSST, a component of the Wechsler Adult Intelligence Test and a test of visuospatial and motor speed-ofprocessing, has a considerable executive function component and is frequently used as a sensitive measure of frontal lobe executive functions [17]. The DSST was used to assess participant cognitive function tasks of pairing (each digit, 1-9, has a symbol it is associated with) and information processing. Participants were asked to 
Table 1. Weighted characteristics of the study variables among a national sample of adults with and without an elevated gamma gap according to 1999-2002 NHANES data

\begin{tabular}{|c|c|c|c|}
\hline Variables & Normal gamma gap $(n=1,230)$ & Elevated gamma gap $(n=1,121)$ & $p$ value \\
\hline Mean DSST & $50.1(48.5-51.7)$ & $44.8(42.9-46.0)$ & $<0.001$ \\
\hline Mean age, years & $69.5(68.94-70.13)$ & $70.4(69.58-71.27)$ & 0.03 \\
\hline Mean A1C level, \% & $5.6(5.61-5.75)$ & $5.9(5.85-6.0)$ & $<0.001$ \\
\hline Mean hypertension, \% & $46.0(0.43-0.50)$ & $53.0(0.49-0.51)$ & 0.01 \\
\hline White race, $\%$ & $89.7(0.87-0.92)$ & $75.0(0.69-0.81)$ & $<0.001$ \\
\hline Current smoker, \% & $12.0(0.10-0.13)$ & $14.0(0.10-0.13)$ & 0.22 \\
\hline Meeting MVPA guidelines, $\%$ & $42.0(0.36-0.47)$ & $31.0(0.26-0.35)$ & $<0.001$ \\
\hline Overweight/obese, $\%$ & $69.0(0.64-0.73)$ & $72.0(0.68-0.76)$ & 0.18 \\
\hline
\end{tabular}

Values are point estimates with 95\% CI in parentheses. A1C, glycated hemoglobin; DSST, Digit Symbol Substitution Test; MVPA, moderate-to-vigorous physical activity.

draw as many symbols as possible that were paired with numbers within $2 \mathrm{~min}$. Following the standard norm-referenced scoring method, one point is given for each correctly drawn and matched symbol, and one point is subtracted for each incorrectly drawn and matched symbol, with a maximum score of 133 .

\section{Physical Activity}

As described elsewhere [18], participants were asked openended questions about participation in leisure-time physical activity over the previous 30 days. Data was coded into 48 activities, including 16 sports-related activities, 14 exercise-related activities, and 18 recreational-related activities. For each activity, Metabolic Equivalent of Task (MET)-min-month was calculated by multiplying the number of days, by the mean duration, by the respective MET level (MET-min-month = days $\bullet$ duration $\bullet$ MET level). Those at or above 2,000 MET-min-month (equivalent to $150 \mathrm{~min} /$ week) were defined as physically active.

\section{Covariates}

For all models, covariates included age, gender, race/ethnicity (Mexican American, other Hispanic, non-Hispanic white, nonHispanic black, other), self-reported smoking (yes/no), energy intake (kcal, continuous), physician-diagnosed hypertension (yes/ no), weight status (overweight/obese [measured body mass index or higher] vs. normal weight), and glycated hemoglobin (\%, continuous).

Participants were classified as smokers if they self-reported smoking every day or some days; otherwise, they were classified as nonsmokers. Previous research demonstrates evidence of validity for self-reported smoking assessment [19]. Energy intake (kcal) was assessed from the mobile examination center interview, per NHANES guidelines, and the follow-up telephone interview. The average of these values was used. If data were missing from the telephone interview, only energy intake obtained from the mobile examination center was used. Glycated hemoglobin was determined from the Primus instrument, which is a fully automated glycohemoglobin analyzer that utilizes the principle of boronate affinity high-performance liquid chromatography.

Physical Activity and Cognitive Function in Older Adults
Analysis

All statistical analyses, computed in Stata (v. 12), accounted for the complex survey design employed in NHANES. An adjusted Wald test was used to examine statistical differences for continuous variables among those with and without an elevated gamma gap (Table 1). A design-based likelihood ratio test was used for categorical variables. A weighted multivariable linear regression model was used to examine the association of meeting physical activity guidelines ( $\geq 2,000 \mathrm{MET}$-min-month) and cognitive function (outcome variable). Statistical significance was established as an alpha $<0.05$.

\section{Results}

The analyzed sample included 2,352 older adults (6085 years). Among these, 1,121 had an elevated gamma gap (3.1-6.3 g/dL) and 1,230 did not (2.0-3.0 g/dL). Characteristics of the study variables, stratified by the gamma gap status, are displayed in Table 1 . Those with an elevated gamma gap, compared to those without, had a worse DSST score (44.8 vs. 50.1 ). This was even further accentuated among those with a more severe gamma gap. For example, when comparing those with a gamma gap level of $>4.0 \mathrm{~g} / \mathrm{dL}$ to those with a level of $<3.0 \mathrm{~g} / \mathrm{dL}$, respectively, the mean DSST scores were 35.7 versus 50.1 . As shown in Table 1, other differences emerged when comparing those with and without an elevated gamma gap. Those with an elevated gamma gap were older, had a lower energy intake, had a higher A1C level, were more likely to be hypertensive, were more likely to be a minority, were more likely to be female, and were less likely to meet the MVPA guidelines. Notably, there were no differences in 
Table 2. Association between meeting physical activity guidelines and cognitive function among those with and without an elevated gamma gap

\begin{tabular}{|c|c|c|c|c|c|c|}
\hline & \multicolumn{3}{|c|}{ Normal gamma gap $(n=1,230)$} & \multicolumn{3}{|c|}{ Elevated gamma gap $(n=1,121)$} \\
\hline & $\beta$ & $95 \% \mathrm{CI}$ & $p$ value & $\beta$ & $95 \% \mathrm{CI}$ & $p$ value \\
\hline $\begin{array}{l}\text { Meeting MVPA guidelines vs. not } \\
\text { Covariates }\end{array}$ & 5.40 & $3.10,7.71$ & $<0.001$ & 6.42 & $3.85,8.98$ & $<0.001$ \\
\hline Age, 1-year increase & -1.0 & $-1.11,-0.9$ & $<0.001$ & -0.78 & $-0.91,-0.64$ & $<0.001$ \\
\hline Female vs. male & 5.27 & $2.71,7.84$ & $<0.001$ & 5.05 & $2.11,8.00$ & 0.001 \\
\hline \multicolumn{7}{|l|}{ Race/ethnicity } \\
\hline Mexican American vs. white & -14.5 & $-18.1,-10.9$ & $<0.001$ & -15.2 & $-19.0,-11.5$ & $<0.001$ \\
\hline African American vs. white & -16.4 & $-20.8,-12.0$ & $<0.001$ & -14.4 & $-17.3,-11.6$ & $<0.001$ \\
\hline Other vs. white & -10.2 & $-16.5,-3.9$ & 0.003 & -12.4 & $-18.2,-6.7$ & $<0.001$ \\
\hline Nonsmoker vs. smoker & 1.3 & $-1.7,4.4$ & 0.37 & 5.0 & $1.3,8.6$ & 0.009 \\
\hline Energy intake, 1-kcal increase & 0.001 & $0.00007,0.003$ & 0.04 & 0.001 & $-0.0005,0.003$ & 0.14 \\
\hline A1C level, $1 \%$ increase & -2.2 & $-3.2,-1.3$ & $<0.001$ & -1.3 & $-2.3,-0.43$ & 0.006 \\
\hline Overweight/obese vs. not & 0.29 & $-2.7,3.3$ & 0.84 & 0.16 & $-3.0,3.3$ & 0.91 \\
\hline Hypertension vs. not & -2.3 & $-4.2,-0.5$ & 0.01 & -2.8 & $-5.9,0.16$ & 0.06 \\
\hline
\end{tabular}

Two multivariable linear regression models were computed: one for those with a normal gamma gap $(<3.1 \mathrm{~g} / \mathrm{dL})$ and one for those with an elevated gamma gap $(\geq 3.1 \mathrm{~g} / \mathrm{dL})$. In both models, covariates included age, gender, race/ethnicity, smoking status, energy intake, A1C level, weight status, and hypertension status. MVPA, moderate-to-vigorous physical activity.

smoking status and weight status among those with and without an elevated gamma gap.

Table 2 displays the weighted multivariable linear regression results among those with and without an elevated gamma gap. Those meeting the MVPA guidelines and not having an elevated gamma gap had a DSST score of 5.4 units higher than their counterparts not meeting the MVPA threshold ( $\beta=5.40,95 \%$ CI 3.10-7.71, $p<0.001$ ). Those meeting the MVPA guidelines and having an elevated gamma gap had a DSST score 6.42 units higher than their counterparts not meeting the MVPA threshold ( $\beta=$ $6.42,95 \%$ CI $3.85-8.98, p<0.001$ ).

\section{Discussion}

The gamma gap is an important clinical measure, as it is useful in the differential diagnosis of chronic diseases associated with physical inactivity and inflammation [7]. The deleterious inflammatory consequences of an elevated gamma gap may potentially influence both physical and cognitive parameters. Previous studies have examined the effects of an elevated gamma gap on cognitive function. Klimkowicz et al. [20] found that increased serum $\gamma$-globulin concentrations are an independent hematological marker of cognitive decline among elderly, prestroke dementia patients. Moreover, expansion of the gamma gap may be a function of senescence. Studies on mice and nonhuman primates suggest that autoimmunity may be proliferated in old age, contributing to progressive cognitive dysfunction via neural degeneration [21]. In this context, autoimmune responses are stimulated by globulin-mediated antibody formation [22]. These antibodies appear to have an increased affinity to neuronal binding sites within the aging brain [23]. This evidence is a cause for concern, as health status, quality of life, and all-cause mortality may be significantly diminished by the risks associated with an elevated gamma gap.

To our knowledge, no study has previously evaluated the direct association between the gamma gap and cognitive function and whether physical activity is favorably associated with cognitive function among those with an elevated gamma gap. However, many studies have examined potential relationships between physical activity and cognitive function [24-28]. Hamer and Chida [26] identified 16 prospective studies that investigated the proposed inverse association between physical activity and cognitive decline. Various analyses point to regular physical activity as a crucial mediator in controlling blood pressure, cholesterol, and the accumulation of proinflammatory cytokines [29], which may impede neural signaling pathways $[26,27]$. Therefore, our present study adds to the research and supplies a clearly defined explanation of physical activity frequency and duration, which has 
been lacking in previous experiments. We found that adequate physical activity levels may attenuate negative neurocognitive outcomes among older individuals with an elevated gamma gap. Indeed, our results demonstrate that those with an elevated gamma gap had worse cognitive function when compared to those with a normal gamma gap. Furthermore, those with an elevated gamma gap who met physical activity guidelines had greater cognitive function when compared to their less active counterparts.

The need for tailored physical activity programs for older adults is of critical importance. Modifiable risk factors for morbidity and mortality increase with age and are manifest among older populations [30, 31]. Moreover, the globulin fraction has been shown to increase in old age [32], while optimal cognitive function may be impaired [33]. Our results are suggestive of a possible inverse relationship between age-associated increases in the gamma gap and insufficient physical activity levels. Furthermore, our findings demonstrate a positive association between physical activity and cognitive function among those with an elevated gamma gap. We have also demonstrated that older age, race/ethnicity minority status, hypertension, and A1C level increase were significantly associated with lower cognitive function among individuals with a normal gamma gap, as well as those with an elevated gamma gap. Female gender and increased energy intake were significantly associated with improved cognitive functioning for those with a normal gamma gap, while female gender and nonsmoking status were significantly related to improved cognition among participants with an elevated gamma gap. Collectively, these results are of clinical importance for medical professionals and exercise physiologists aiming to improve physical and mental outcomes for the elderly.
This study is not without limitations, which include the subjective assessment of physical activity and crosssectional design. In addition, this study utilized participant responses to a single cognitive task (DSST), which may not necessarily be generalizable to other cognitive domains. Cognitive impairment associated with an elevated gamma gap in older adults may also limit the validity of self-report data although we do not believe that a mild level of cognitive impairment associated with aging would affect the ability of participants to accurately respond to interview questions. Nevertheless, there is a possibility that this level of cognitive dysfunction may attenuate self-report accuracy for some aging individuals evaluated for this study. Notable strengths include the national sample and study novelty. Thus, future prospective work on this topic using an objective measure of physical activity is warranted.

\section{Conclusions}

This study has important clinical implications, as the gamma gap may be predictive of the risk for early morality and reduced quality of life. Future work is also needed to examine the extent to which the observed differences in cognition in the present sample are clinically meaningful differences. Clinicians and health professionals may look at prescribing safe and tailored physical activity participation as a potential strategy to offset or mitigate the deleterious effects of gamma gap inflation during older age. However, experimental work is needed to investigate whether physical activity training programs are efficacious in meaningfully reducing an elevated gamma gap and preserving optimal cognitive functioning among atrisk individuals.

\section{References}

1 Jain KK. Personalized Management of Cardiovascular Disorders. Medical principles and practice : international journal of the Kuwait University. Health Science Centre. 2017; 26:399-414.

2 Loprinzi PD, Addoh O, Joyner C. Multimorbidity, mortality, and physical activity. Chronic Illn. 2016 Dec;12(4):272-80.

3 Busher JT. Serum Albumin and globulin. In: Walker HK, Hall WD, Hurst JW, editors. Clinical methods: the history, physical, and laboratory examinations. 3rd ed. Boston: Butterworth; 1990.
4 Eckersall PD. Proteins, proteomics, and the dysproteinemias. In: Clinical biochemistry of domestic animals. 6th ed. 2008. p. 117-155.

5 Juraschek SP, Moliterno AR, Checkley W, Miller ER 3rd. The Gamma Gap and AllCause Mortality. PLoS One. 2015 Dec; 10(12):e0143494.

6 Yaffe K, Kanaya A, Lindquist K, Simonsick EM, Harris T, Shorr RI, et al. The metabolic syndrome, inflammation, and risk of cognitive decline. JAMA. 2004 Nov;292(18):223742.
7 Edwards MK, Loprinzi PD. Atherogenic index of plasma and the gamma gap: considerations by physical activity. Int J Cardiol. 2016 Nov;222:946-8.

8 Loprinzi PD, Addoh O. The gamma gap and all-cause mortality risk: considerations of physical activity. Int J Clin Pract. 2016 Jul; 70(7):625-9.

9 Gopal DM, Kalogeropoulos AP, Georgiopoulou VV, Tang WW, Methvin A, Smith AL, et al.; Health ABC Study. Serum albumin concentration and heart failure risk The Health, Aging, and Body Composition Study. Am Heart J. 2010 Aug;160(2):279-85. 
10 Mehlig K, Skoog I, Waern M, Miao Jonasson J, Lapidus L, Björkelund C, et al. Physical activity, weight status, diabetes and dementia: a 34-year follow-up of the population study of women in Gothenburg. Neuroepidemiology. 2014;42(4):252-9.

11 Gielen S, Adams V, Linke A, Erbs S, MöbiusWinkler S, Schubert A, et al. Exercise training in chronic heart failure: correlation between reduced local inflammation and improved oxidative capacity in the skeletal muscle. Eur J Cardiovasc Prev Rehabil. 2005 Aug;12(4): 393-400.

12 Pierce TW, Madden DJ, Siegel WC, Blumenthal JA. Effects of aerobic exercise on cognitive and psychosocial functioning in patients with mild hypertension. Health Psychol. 1993 Jul;12(4):286-91.

13 American College of Sports Medicine. ACSM's guidelines for exercise testing and prescription. Lippincott Williams \&; Wilkins; 2013.

14 Loprinzi PD. Multimorbidity, cognitive function, and physical activity. Age (Dordr). 2016 Feb;38(1):8.

15 Loprinzi PD. Epidemiological investigation of muscle-strengthening activities and cognitive function among older adults. Chronic Illn. 2016 Jun; 12(2):157-62.

16 Loprinzi PD, Crush E. Physical activity and cognitive function among older adults with congestive heart failure patients. J Molecul Pathophysiol. 2016;5(2):28-30.

17 Nakahachi T, Ishii R, Iwase M, Canuet L, Takahashi H, Kurimoto R, et al. Frontal activity during the digit symbol substitution test determined by multichannel near-infrared spectroscopy. Neuropsychobiology. 2008; 57(4):151-8.
18 Loprinzi PD. Dose-response association of moderate-to-vigorous physical activity with cardiovascular biomarkers and all-cause mortality: considerations by individual sports, exercise and recreational physical activities. Prev Med. 2015 Dec;81:73-7.

19 Yeager DS, Krosnick JA. The validity of selfreported nicotine product use in the 20012008 National Health and Nutrition Examination Survey. Med Care. 2010 Dec;48(12): 1128-32.

20 Klimkowicz A, Dziedzic T, Polczyk R, Pera J, Słowik A, Szczudlik A. Factors associated with pre-stroke dementia. J Neurol. 2004;251: 599-603.

21 Varnum MM, Ikezu T. The classification of microglial activation phenotypes on neurodegeneration and regeneration in Alzheimer's disease brain. Arch Immunol Ther Exp (Warsz). 2012 Aug;60(4):251-66.

22 Nandy K. Brain-reactive antibodies in sera of aging non-human primates. Mech Ageing Dev. 1981 Jun;16(2):141-7.

23 Ingram CR, Phegan KJ, Blumenthal HT. Significance of an aging-linked neuron binding gamma globulin fraction of human sera. J Gerontol. 1974 Jan;29(1):20-7.

24 Lautenschlager NT, Cox KL, Flicker L, Foster JK, van Bockxmeer FM, Xiao J, et al. Effect of physical activity on cognitive function in older adults at risk for Alzheimer disease: a randomized trial. JAMA. 2008 Sep;300(9):102737.

25 Loprinzi PD, Wolfe CD, Walker JF. Exercise facilitates smoking cessation indirectly via improvements in smoking-specific self-efficacy: prospective cohort study among a national sample of young smokers. Prev Med. 2015 Dec;81:63-6.
26 Hamer M, Chida Y. Physical activity and risk of neurodegenerative disease: a systematic review of prospective evidence. Psychol Med. 2009 Jan;39(1):3-11.

27 Cotman CW, Berchtold NC, Christie LA. Exercise builds brain health: key roles of growth factor cascades and inflammation. Trends Neurosci. 2007 Sep;30(9):464-72.

28 Lee KS, Lee Y, Back JH, Son SJ, Choi SH, Chung YK, et al. Effects of a multidomain lifestyle modification on cognitive function in older adults: an eighteen-month communitybased cluster randomized controlled trial. Psychother Psychosom. 2014;83(5):270-8.

29 Wahlin-Larsson B, Wilkinson DJ, Strandberg E, Hosford-Donovan A, Atherton PJ, Kadi F. Mechanistic links underlying the impact of Creactive protein on muscle mass in elderly. Cell Physiol Biochem. 2017;44:267-278.

30 Ngandu T, Lehtisalo J, Solomon A, Levälahti E, Ahtiluoto S, Antikainen R, et al. A 2 year multidomain intervention of diet, exercise, cognitive training, and vascular risk monitoring versus control to prevent cognitive decline in at-risk elderly people (FINGER): a randomised controlled trial. Lancet. 2015 Jun; 385(9984):2255-63.

31 Chatterji S, Byles J, Cutler D, Seeman T, Verdes E. Health, functioning, and disability in older adults - present status and future implications. Lancet. 2015 Feb;385(9967):56375.

32 Woodford-Williams E, Alvarez AS, Webster D, Landless B, Dixon MP. Serum protein patterns in 'normal'and pathological ageing. Gerontology. 1964;10(2-3):86-99.

33 Hedden T, Gabrieli JD. Insights into the ageing mind: a view from cognitive neuroscience. Nat Rev Neurosci. 2004 Feb;5(2):87-96. 